\title{
GPC and Holo-GPC for laser beam shaping in 2D and 3D
}

\author{
Glückstad, Jesper; Bañas, Andrew Rafael
}

\section{Published in:}

Proceedings of SPIE

Link to article, DOI:

$10.1117 / 12.2323369$

Publication date:

2018

Document Version

Publisher's PDF, also known as Version of record

Link back to DTU Orbit

Citation (APA):

Glückstad, J., \& Bañas, A. R. (2018). GPC and Holo-GPC for laser beam shaping in 2D and 3D. In Proceedings of SPIE (Vol. 10744). [107440G ] SPIE - International Society for Optical Engineering. Proceedings of SPIE The International Society for Optical Engineering https://doi.org/10.1117/12.2323369

\section{General rights}

Copyright and moral rights for the publications made accessible in the public portal are retained by the authors and/or other copyright owners and it is a condition of accessing publications that users recognise and abide by the legal requirements associated with these rights.

- Users may download and print one copy of any publication from the public portal for the purpose of private study or research.

- You may not further distribute the material or use it for any profit-making activity or commercial gain

- You may freely distribute the URL identifying the publication in the public portal 


\section{GPC and Holo-GPC for laser beam shaping in 2D and $3 D$}

\section{Jesper Glückstad, Andrew Bañas}

Jesper Glückstad, Andrew Bañas, "GPC and Holo-GPC for laser beam shaping in 2D and 3D," Proc. SPIE 10744, Laser Beam Shaping XVIII, 107440G (14 September 2018); doi: 10.1117/12.2323369

SPIE Event: SPIE Optical Engineering + Applications, 2018, San Diego, California, United States 


\title{
GPC and Holo-GPC for laser beam shaping in 2D and 3D
}

\author{
Jesper Glückstad ${ }^{1,2}$ and Andrew Bañas ${ }^{1,2}$ \\ ${ }^{1}$ Technical University of Denmark, DK-2800 Kgs. Lyngby, Denmark \\ ${ }^{2}$ OptoRobotix ApS, DK-2000 Frederiksberg, Denmark \\ email: jesper.gluckstad@fotonik.dtu.dk \\ www.ppo.dk www.GPCphotonics.com www.OptoRobotix.com
}

\begin{abstract}
We have invented an efficient phase-only light shaping modality that can simultaneously control the distribution of multiple beams and shaping of these beams individually in a volume. It is coined Holo-GPC and extends the capabilities of both Generalized Phase Contrast (GPC) and Holography. Holo-GPC can be considered as a hybrid combination of holography that can create extended 2D or 3D beam distributions and GPC that forms noise-free sculpting of the individual beams.
\end{abstract}

Keywords: Generalized Phase Contrast (GPC), Holographic GPC (Holo-GPC), laser beam shaping

\section{INTRODUCTION}

Laser beam shaping techniques based on phase-only modulation offer multiple advantages over amplitude modulation. This paper discusses and compares the merits of two phase modulation techniques; phase-only computer generated holography and Generalized Phase Contrast (GPC). Both techniques are briefly presented while recent developments in GPC will also be covered. Furthermore, novel hybrid schemes that inherit merits from both holography and GPC are also covered. In particular, our most recent technique coined "Holo-GPC" will be discussed in addition to earlier hybrid techniques. We will discuss how Holo-GPC utilizes the simplicity of GPC in forming well-defined speckle-free shapes and the versatility of holography in distributing these shaped beams over an extended 3D volume. To conclude, we cite applications where the combined strengths of the two photon-efficient phase-only light shaping techniques open new possibilities.

\section{GENERALIZED PHASE CONTRAST (GPC)}

Generalized Phase Contrast method (GPC), belongs to a class of non-absorbing common path interferometers [1]. A phase transmittance distribution directly representing the desired output intensity is imaged through the interference with a reference wave synthesized from phase-shifted low spatial frequencies. This is implemented using a phase contrast filter (PCF) whose phase shifting region is situated at the center of the optically Fourier transformed input. Static GPC light shapers have been implemented with binary phase plates and have been used for efficiently illuminating spatial light modulators (SLM) [2]. Likewise, dynamic GPC is implemented using a programmable phase-only SLM and has been used in experiments such as optical trapping, two-photon excitation and neurophotonics [3].

Unlike diffractive approaches, GPC uses the target shape as the phase mask that directly interfaces with the incident Gaussian, instead of a pattern based on the target's Fourier transform (e.g. holograms). This makes GPC robust to input beam variations as convolution or other spatial filtering effects are avoided. The use of a common path configuration renders steep well-defined edges in the shaped output. Furthermore, the target output shapes could easily be replaced through interchangeable phase masks or by simply redrawing the pattern encoded into the SLM.

Laser Beam Shaping XVIII, edited by Angela Dudley, Alexander V. Laskin, Proc. of SPIE Vol. 10744 107440G · @ 2018 SPIE · CCC code: 0277-786X/18/\$18 · doi: 10.1117/12.2323369 


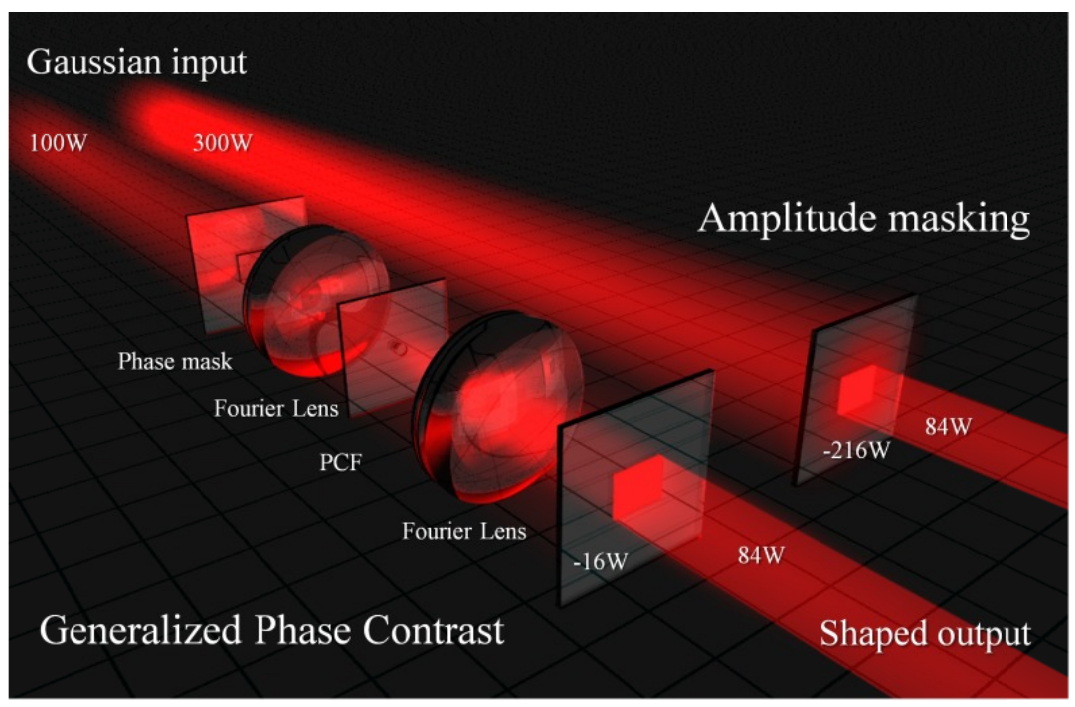

Figure 1. GPC-system efficiently transforming an incident Gaussian beam into a bright rectangle. Besides a standard telescopic $4 f$ setup formed by the two Fourier lenses, GPC uses a simple binary phase mask at the input and phase contrast filter at the Fourier plane. An amplitude masking configuration is shown besides to illustrate the significant difference in energy utilization when producing the same shaped output (Adapted from ref. [4]).

GPC's use of an imaging geometry makes it advantageous with techniques that use multiple wavelengths, pulsed or broadband sources. One example is temporal focusing which effectively confines light along the axial direction and is therefore relevant in printing 2D slices in Laser Direct Writing or for exciting neurons in two-photon based optogenetics experiments [3]. Temporal focusing temporarily stretches a pulsed light source such that the intensity is lowered except at the focal plane. This means for creating isolated speckle-free "light sheets" have been used for two-photon excitation of selected neurons at an isolated plane within the tissue volume. If the biological tissue were instead replaced by photo-resist, GPC combined with temporal focusing would be able to solidify a 2D slice without affecting the planes adjacent to that slice.
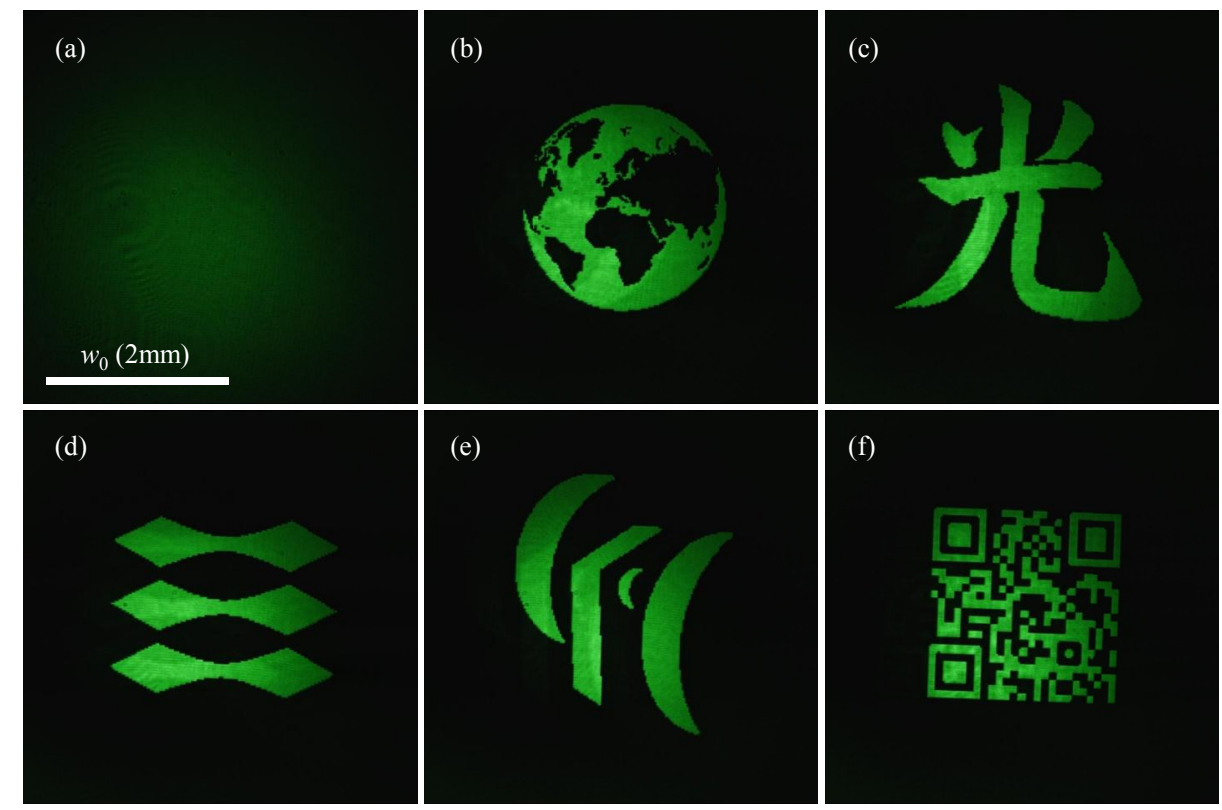

Figure 2. GPC-generated intensity patterns obtained from an expanded green laser beam. Phase patterns are optimally scaled and then drawn on a phase-SLM and illuminated with the Gaussian beam imaged in (a). Adapted from ref. [4). 

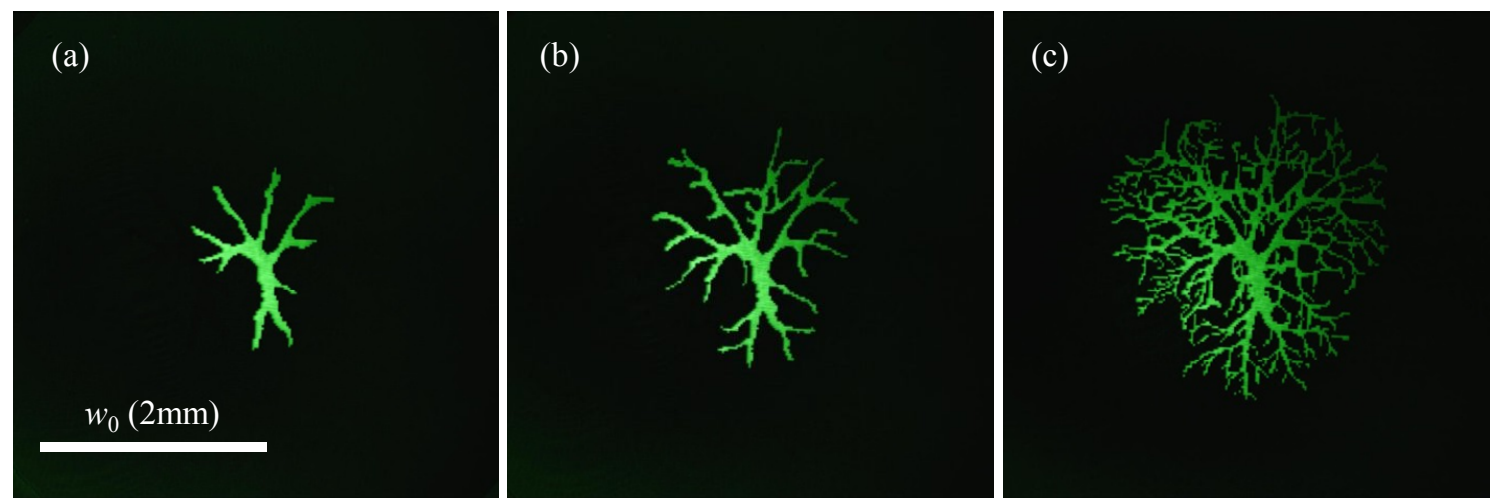

Figure 3. Dynamically evolving GPC-generated patterns from a green laser. Phase patterns of neurons are optimally scaled and then drawn on a phase-SLM and illuminated with the Gaussian beam imaged in (a). Adapted from ref. [4].

We have previously shown theoretically [5] and experimentally [6] that GPC shows robustness to shift in wavelength and can maintain both projection length scale and high efficiency over a range $\left[0.75 \lambda_{0} ; 1.5 \lambda_{0}\right]$ with $\lambda_{0}$ as the characteristic design wavelength. With the resulting performance across multiple wavelengths and the recent availability of tabletop super-continuum lasers, GPC light shaping opens the possibility for creatively incorporating various multi-wavelength approaches into spatially shaped excitations that can enable new broad-band light applications.

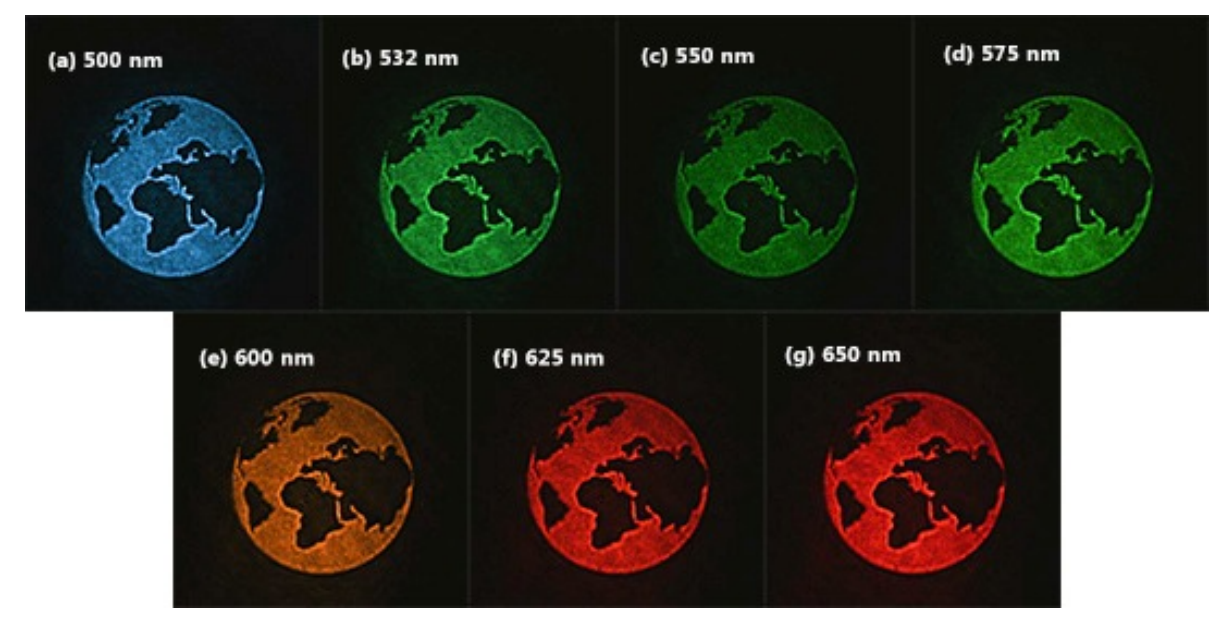

Figure 4. Color CCD images of GPC-projections using a supercontinuum laser. The power at different wavelengths are adjusted individually for visibility. The input Gaussian beam size exhibits some wavelength dependence. Adapted from ref. [6].

\section{HOLOGRAPHIC GPC (HOLO-GPC)}

We have previously shown how to modify a GPC-setup to produce output spots instead of the mapped phase images by using matched filtering $[7,8]$. In addition to the phase shifting circular region at the PCF, concentric phase shifting rings are applied to the Fourier distribution to match the alternating lobes of the Airy disk that correspond to specified input circles at the input phase mask. This modified GPC embodiment, consisting of a matched filter at the Fourier plane, is thus called matched filtering GPC (mGPC). 
While being relatively simple to implement, mGPC does not fully exploit the benefits of digital holography. Both GPC and mGPC, only have a fixed element at the Fourier plane which is the PCF or a matched filter. This fixed Fourier filter makes both of them practical to implement requiring only a single SLM to dynamically reconfigure the output. The fixed matched filter in mGPC also limits the features that it can borrow from $2 f$ digital holography. In a typical $2 f$ holographic setup, a dynamic SLM is behind the Fourier transforming lens. In GPC or mGPC, the $2 f$ part defined by the region between the PCF or matched filter and the output beams, on the other hand, has a " $2 f$ input" that is not as dynamic as an illuminated SLM.

As suggested by general Fourier optics considerations, there are far more possibilities if the phase element at the Fourier plane can also be dynamically controlled. Indeed, near ideal arbitrary complex fields can be generated when using a tandem of two dynamic SLM surfaces. Hence, recently we have developed "HoloGPC" that takes advantage of some features of a so-called "tandem configuration" by having a phase modulator at the spatial frequency or Fourier plane. However, similar to GPC and mGPC, Holo-GPC maintains its practicality of requiring just one active SLM. Thus, to maintain this practicality and economy, the first phase modulating element is a well defined, easily fabricated, and generally reusable phase mask instead of another SLM as the tandem configuration suggests. In the usual tandem configuration, the first phase element being a hologram generally bears no resemblance to the final output and would require re-calculation for different outputs. Pre-fabricated phase elements would be impractical as they cannot be specified with a few parameters, require multiple phase levels to be efficient and would have to be replaced for each new output configuration.

Just as the shape of the generated individual output beams can be important, so is the shape of the light that is illuminating a hologram. Ultimately, the read-out illumination determines the amplitude and phase distribution of the point-spread function (PSF) at the output optical far-field plane. We are particularly interested in modifying the "spread function" of the output beams. As the typical illumination shape would have a tophat or a Gaussian distribution, the typical PSFs are either Airy-disk or Gaussian shaped. The target output PSF can thus be changed by illuminating with an (inverse) far-field transformed beam shape. The challenge, therefore, is the efficient creation of an initial basis beam shape (typically using the Fourier transform) that will become the output's PSF.

It is well known that a Fourier transformed amplitude mask can be used to illuminate a hologram in order to get PSFs with the same amplitude pattern. Holo-GPC starts with a similarly looking phase mask that efficiently transmits the input light without absorbing photons. But unlike a straightforward convolution, phase filtering is required to convert the phase mask into shaped PSFs at the output. As it relies on GPC's phase to intensity mapping, Holo-GPC also inherits GPC's efficiency advantage over amplitude masking and would in principle also have $3 \mathrm{x}$ brighter PSFs or over $90 \%$ of energy savings.

To understand how Holo-GPC works, we first briefly revisit the standard GPC configuration and subsequently identify the modifications necessary to make multiple holographic copies of this GPC output. In a standard GPC setup (i.e. Figure 5), the input phase mask is first optically Fourier transformed and the resulting distribution is focused on a phase contrast filter (PCF). An output intensity mapping of the input phase mask is generated from the interference of the imaged input with a so-called synthesized reference wave (SRW) that results from the low frequency components phase shifted by the PCF.

We treat the region between the PCF plane, through the imaging lens, then to the output intensity (Fig. 5(d) to (g)), as a Fourier transforming configuration. The optical Fourier transform of the input phase mask can be thought of as the illumination at this plane. For a rectangular or circular input phase mask, this PCF illumination typically resembles a Sinc-function, or an Airy disk, but in general, this can be the Fourier transform of the desired PSF-shape with similar geometry as the input phase mask. As with holography, the PCF's phase shift is correcting the central part, such that it matches the phase distribution of an ideal Sinc function, Airy disk or Fourier transform of the desired PSF pattern. Hence, through convolution, by placing a hologram phase on top of this PCF-shifted, Fourier-transformed phase mask, Holo-GPC can produce a beam 
distribution wherein each beam takes the form of the intensity mapped input illuminated phase mask as in Figure 6.

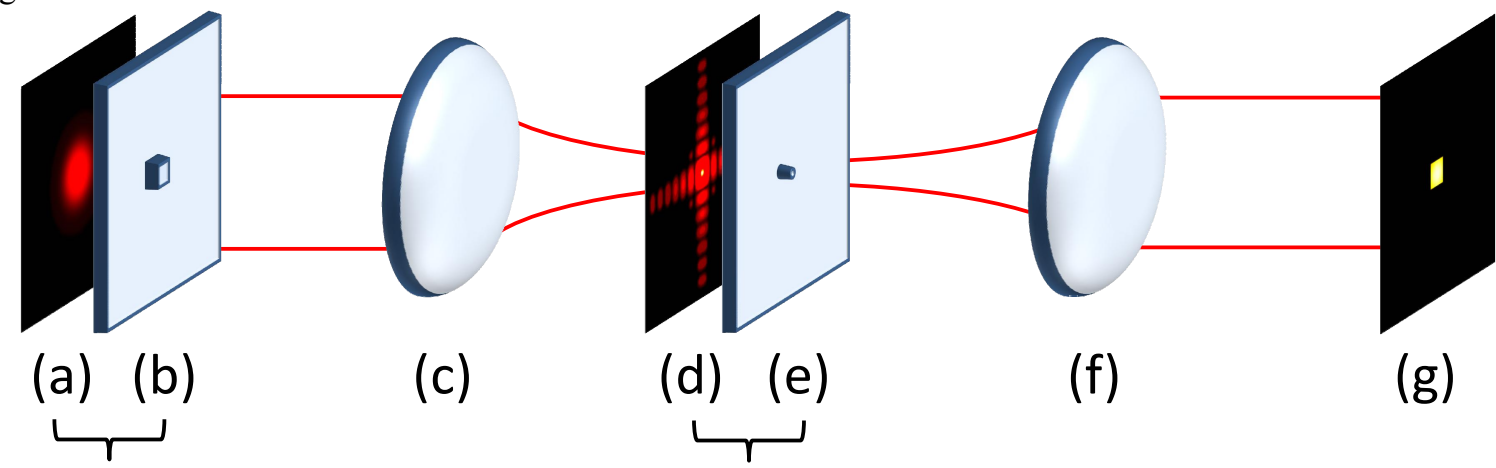

Figure 5. Standard GPC setup consisting of an input phase mask (b), Fourier lenses (c) \& (f) and a PCF (e). The input illumination (a), its Fourier transform, after applying the phase mask (d) and the imaged intensity (g) are also shown. Adapted from ref. [10].

As Holo-GPC operates by efficiently modifying the point spread function, the individual beams are identical copies of the intensity-imaged phase mask pattern. This multi-beam approach is a different paradigm from standard GPC wherein a phase mask utilizes multiple smaller sub-shapes that need not be identical, and hence the corresponding output individual beams can also have different shapes. However, unlike standard GPC or mGPC, Holo-GPC's output beams are not constrained to an imaging plane, but rather, can be addressed in a $3 \mathrm{D}$ holographic manner. Furthermore, a compensating phase mask region is not necessary for maintaining the optimal fill factor while changing the number of output beams.

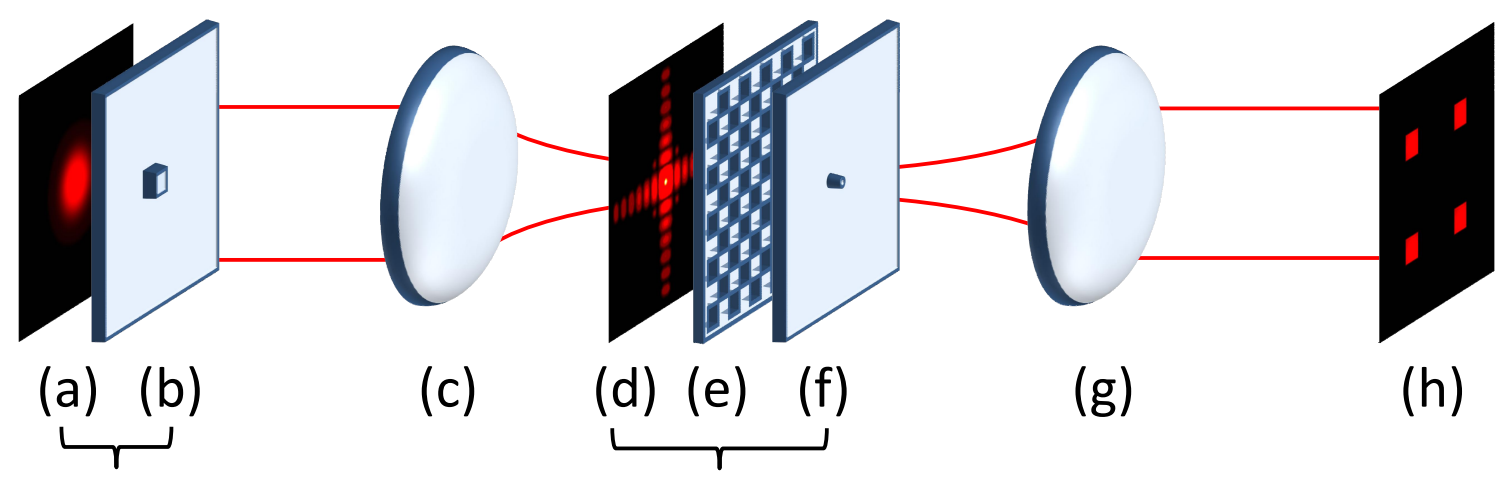

Figure 6. Holo-GPC setup. Compared to standard GPC, a hologram (e) is placed in addition to the PCF at the Fourier plane of the first lens. For practical implementations, the hologram is typically encoded on an SLM, and the sizes of the input beam, phase mask, PCF and focal lengths have to be adjusted. The second lens (g) optically Fourier transforms the light that is altered by the hologram and PCF to get a distributed output consisting of specklefree contiguous shapes. Adapted from ref. [10]).

\section{HOLO-GPC EXPERIMENTS}

To demonstrate Holo-GPC experimentally we used a $\lambda=750 \mathrm{~nm}$ laser source (filtered super-continuum laser from NKT Photonics) and re-purposed a static GPC Light Shaper (LS) from earlier experiments [4, 9]. The PCF within the static GPC-LS was displaced from the beam path, hence, switching off the standard GPC operation. An 8x magnified image of the GPC-LS's focal plane was used to illuminate a phase-only SLM (Hamamatsu Photonics, pitch $=20 \mu \mathrm{m}$ ). The SLM-encoded PCF shifting region had a diameter of 21 pixels or $420 \mu \mathrm{m}$ which is very close to the theoretical optimal $423.26 \mu \mathrm{m}$. The complex field at the SLM plane was 
optically Fourier transformed with an $f=300 \mathrm{~mm}$ lens, then magnified for sufficient coverage of the CCD camera.
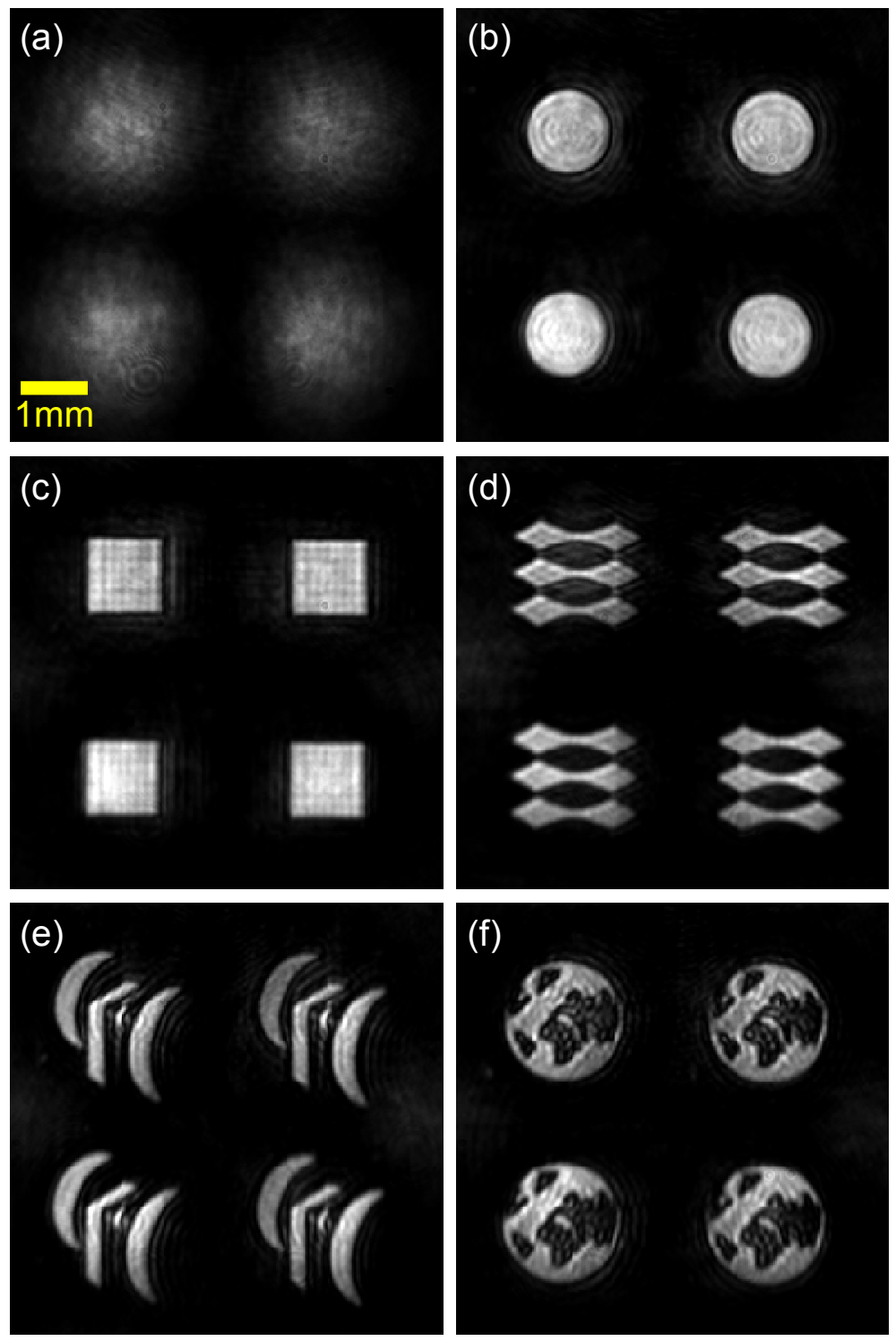

Figure 7. Initial holographic output from a checkerboard grating (a), then with Holo-GPC encoded with different phase masks (b)-(f). The scale bar corresponds to $1 \mathrm{~mm}$ at the camera. Adapted from ref. [10].

For the holograms, we tested a simple binary checkerboard grating and multiplexed spots distributed in 3D. The multiplexed spots were based on the "lenses and prisms" phases encoded on non-overlapping random SLM regions that are assigned to each spot. For visualization, a blazed grating was used to shift the spot patterns away from the zero-order diffraction. Uniform illumination was used in the CGH calculations. For a given CGH, different phase masks were used to form different PSF shapes.

The output reconstructions from the binary checkerboard grating using different input phase masks are shown in Figure 7. Some loss in sharpness and fringing can be attributed to the finite SLM window and lens apertures, but the input patterns remain recognizable. The same SLM hologram is used even for the arbitrarily shaped 
phase masks. Figure 8 demonstrates 3D addressing with Holo-GPC by using a multi-spot hologram and then imaging the reconstructed output at planes by translating the camera by $215 \mathrm{~mm}$. Despite some noise from the hologram among the individual beams, it is clear that the square mask is focused at different planes and exhibits expected diffraction patterns at the off-focus planes.
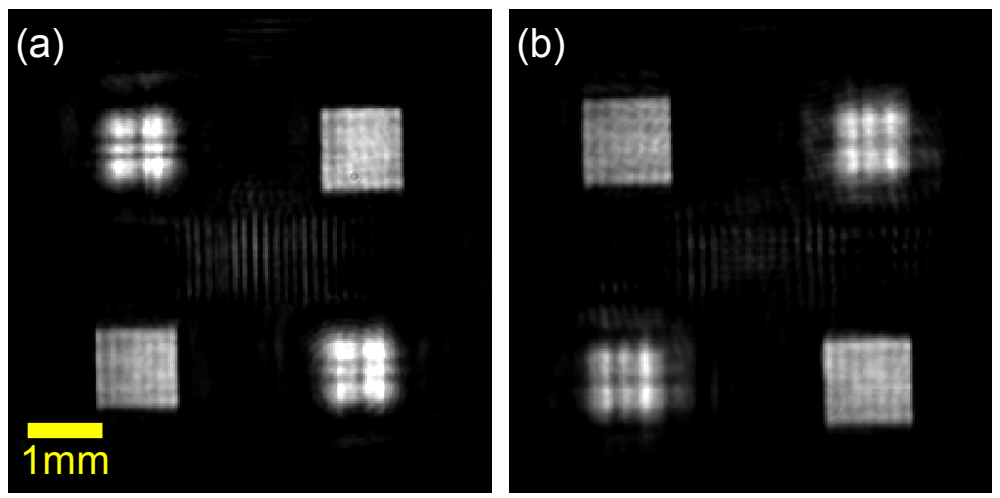

Figure 8. Holo-GPC addressing beams in 3D. Planes in (a) and (b) are $215 \mathrm{~mm}$ apart along the axial direction and include both "in focus" and diffracted "out-of-focus" square beam reconstructions. Adapted from ref. [10].

\section{REFERENCES}

[1] Glückstad, J.., Palima, D. Z., "Generalized Phase Contrast: Applications in Optics and Photonics," Springer Series in Optical Sciences (2009).

[2] Villangca, M., Bañas, A., Palima, D.., Glückstad, J., "GPC-enhanced read-out of holograms," Opt. Commun. 351, 121-127 (2015).

[3] Papagiakoumou, E., Anselmi, F., Bègue, A., de Sars, V., Glückstad, J., Isacoff, E. Y.., Emiliani, V., "Scanless two-photon excitation of channelrhodopsin-2," Nature Methods 7(10), 848-854 (2010).

[4] Bañas, A., Kopylov, O., Villangca, M., Palima, D.., Glückstad, J., "GPC Light Shaper: static and dynamic experimental demonstrations," Opt. Express 22, 23759-23769 (2014).

[5] Palima, D.., Glückstad, J., "Multi-wavelength spatial light shaping using generalized phase contrast," Opt. Express 16(2), 1331-1342 (2008).

[6] Kopylov, O., Bañas, A., Villangca, M.., Palima, D., "GPC light shaping a supercontinuum source," Opt. Express 23, 1894-1905 (2015).

[7] Bañas, A., Palima, D., Glückstad, J., "Matched-filtering generalized phase contrast using LCoS picoprojectors for beam-forming," Opt. Express 20, 9705-12 (2012).

[8] Bañas, A., Aabo, T., Palima, D., Glückstad, J., "Matched filtering Generalized Phase Contrast using binary phase for dynamic spot- and line patterns in biophotonics and structured lighting," Opt. Express 21, 388-394 (2013).

[9] Villangca, M., Bañas,A., Palima, D., Glückstad, J., "Dark GPC: extended nodal beam areas from binaryonly phase," Opt. Eng. 55, 125102 (2016).

[10] Bañas, A., Glückstad, J., "Holo-GPC: Holographic Generalized Phase Contrast," Opt. Commun. 392, 190-195 (2017). 